\title{
Abaloparatide-SC improves trabecular microarchitecture as assessed by trabecular bone score (TBS): a 24-week randomized clinical trial
}

\author{
J. P. Bilezikian ${ }^{1}$ - G. Hattersley ${ }^{2}$ - L. A. Fitzpatrick ${ }^{2}$ - A. G. Harris ${ }^{2}$ - E. Shevroja ${ }^{3}$. \\ K. Banks ${ }^{2}$ B. Z. Leder $^{4}$ - J. R. Zanchetta ${ }^{5}$ D. Hans ${ }^{3}$
}

Received: 11 July 2017 / Accepted: 6 November 2017 / Published online: 22 November 2017

(C) The Author(s) 2017. This article is an open access publication

\begin{abstract}
Summary In a phase 2 trial of 222 postmenopausal women with osteoporosis aged 55 to 85 years randomized to one of three different doses of abaloparatide-SC, subcutaneous teriparatide, or placebo for 24 weeks, abaloparatide-SC resulted in improvements in skeletal microarchitecture as measured by the trabecular bone score.

Introduction Subcutaneous abaloparatide (abaloparatideSC) increases total hip and lumbar spine bone mineral density and reduces vertebral and non-vertebral fractures. In this study, we analyzed the extent to which abaloparatideSC improves skeletal microarchitecture, assessed indirectly by trabecular bone score (TBS).

Methods This is a post hoc analysis of a phase 2 trial of 222 postmenopausal women with osteoporosis aged 55 to 85 years randomized to abaloparatide-SC (20, 40, or $80 \mu \mathrm{g})$, subcutaneous teriparatide $(20 \mu \mathrm{g})$, or placebo for 24 weeks. TBS was measured from lumbar spine dual X-ray absorptiometry (DXA) images in 138 women for whom the DXA device
\end{abstract}

Study sponsor: Radius Health, Inc.

G. Hattersley

ghattersley@ radiuspharm.com

1 Department of Endocrinology, Columbia University College of Physicians and Surgeons, New York, NY, USA

2 Radius Health, Inc., Waltham, MA, USA

3 Center of Bone Diseases, Bone and Joint Department, Lausanne University Hospital, Lausanne, Switzerland

4 Department of Medicine, Harvard Medical School, Endocrine Unit, Massachusetts General Hospital, Boston, MA, USA

5 Instituto de Investigaciones Metabólicas, Libertad, 836 Buenos Aires, Argentina was TBS software compatible. Assessments were made at baseline, 12 and 24 weeks. Between-group differences were assessed by generalized estimating equations adjusted for relevant baseline characteristics, and a pre-determined least significant change analysis was performed.

Results After 24 weeks, TBS increased significantly by 2.27 , 3.14 , and $4.21 \%$ versus baseline in participants on 20,40 , and $80 \mu \mathrm{g}$ abaloparatide-SC daily, respectively, and by $2.21 \%$ in those on teriparatide $(p<0.05$ for each). The TBS in the placebo group declined by $1.08 \%$. The TBS increase in each treatment group was significantly higher than placebo at 24 weeks $(p<0.0001$ for each) after adjustment for age, BMI, and baseline TBS. A dose-response was observed at 24 weeks across the three doses of abaloparatide-SC and placebo $(p=0.02)$. The increase in TBS in the abaloparatide-SC $80 \mu \mathrm{g}$ group was significantly greater than TPTD $(p<0.03)$. Conclusions These results are consistent with an effect of abaloparatide-SC to improve lumbar spine skeletal microarchitecture, as assessed by TBS.

Keywords Anabolics · Bone microarchitecture $\cdot$ Clinical trials · Osteoporosis

\section{Introduction}

Abaloparatide, a novel 34-amino acid peptide designed to be a selective activator of the $\mathrm{PTH} 1$ receptor signaling pathway, is a promising therapeutic approach to the treatment of osteoporosis. Abaloparatide binds selectively to the $\mathrm{RG}$ versus $\mathrm{R}^{0}$ conformation of the $\mathrm{PTH} 1$ receptor, resulting in transient receptor signaling consistent with a net anabolic effect [1]. The results of the phase 3 ACTIVE trial of abaloparatide for subcutaneous injection (abaloparatide-SC) demonstrated efficacy in reducing vertebral and non-vertebral fracture incidence [2]. 
We hypothesized that since abaloparatide-SC is an activator of the PTH1 receptor, it is likely that its efficacy is related to improved bone microarchitecture and strength, as has been shown for teriparatide [3, 4]. To this point, animal studies with abaloparatide-SC have documented complete reversal of bone loss in ovariectomy-induced osteopenic rats and monkeys [1, $5,6]$. In monkeys, abaloparatide-SC augments not only bone mass and volume, but also bone microarchitecture and bone strength [7]. In both animal models, the increased bone formation occurs in the absence of a corresponding increase in bone resorption, resulting in marked gains in bone mass and strength $[7,8]$. To date, however, the effects of abaloparatide$\mathrm{SC}$ on trabecular microarchitecture and bone strength have not been assessed in human subjects.

The trabecular bone score (TBS) is a non-invasive imaging technology that takes advantage of the DXA lumbar spine image, providing skeletal information not captured by standard bone mineral density (BMD) assessments. It is a graylevel textural analysis that utilizes experimental variograms to assess 2D projection images from the DXA scan [9]. A semiquantitative measurement, TBS is strongly correlated with a number of microarchitectural parameters that reflect bone strength [10]. A high TBS reflects better microarchitecture; whereas, a low TBS reflects worse microarchitecture [11]. In a recent meta-analysis, TBS was demonstrated to be a significant predictor of fracture risk independent of Fracture Risk Assessment Tool (FRAX)-calculated 10-year fracture risk estimate [12].

The primary objective of this study was to utilize TBS in a six-month clinical trial of several different doses of abaloparatide-SC, a single dose of teriparatide, or placebo [13], to determine the extent to which abaloparatide-SC improves skeletal microarchitecture.

\section{Methods}

This study is a post hoc retrospective analysis of a phase 2, multicenter, international, and double-blind placebo-controlled trial conducted at 30 study centers across four countries: the USA, Argentina, India, and the UK [13]. All patients had provided informed written consent prior to their participation, in accordance with the Declaration of Helsinki for research on human subjects.

To be eligible for inclusion in the original trial, patients had to be postmenopausal women with osteoporosis, aged 55 years old or older, and not currently on long-term treatment for osteoporosis. In addition, patients could not currently be on systemic corticosteroids and could not have any other bone or spinal disease or history of past injuries or surgeries (e.g., total hip replacement, spinal rods) that would alter BMD measurements at the lumbar spine or hip. Once screened and confirmed for eligibility, patients were randomized to one of the following five treatment arms, with each patient selfadministering a single subcutaneous injection once daily for 24 weeks of placebo; one of three different doses of abaloparatide-SC $(20,40$, or $80 \mu \mathrm{g})$; and teriparatide $20 \mu \mathrm{g}$. All patients also received supplemental calcium (500 to $1000 \mathrm{mg}$ ) and vitamin D (400 to $800 \mathrm{IU}$ ), in accordance with local practices. Patients and investigators remained blinded to treatment with all doses of abaloparatide-SC and placebo throughout the study. Blinding was not possible in patients randomized to receive teriparatide because the drug could be delivered only via its trademarked injection pen [13].

BMD was assessed in the lumbar spine, femoral neck, and total hip by DXA at baseline and again at 12 and 24 weeks after the initiation of treatment. DXA scans were sent to a central imaging reader (BioClinica Inc., Doylestown, PA, USA) for a quality control review, followed by analysis in accordance with each manufacturer's instructions and ISCD guidelines. The two followup scans on each given patient were performed on the same instrument used at baseline. Each study site performed instrument quality control assessments over time (i.e., instrument standardization and phantom calibration), which were reviewed by the central reader. Further details on the methodology of the original trial have been published [13]. All vertebrae (L1 to L4) were automatically included into the analysis (about $84 \%$ of all patients). However, individual vertebrae were excluded if fractures or degenerative changes were present, in accordance with ISCD rules for individual vertebrae exclusion (more than 1 standard deviation in $\mathrm{T}$-score from the immediately adjacent vertebrae).

To be eligible for the current study, patients had to have had their initial BMD measurement on a DXA scanner compatible with TBS software. TBS was calculated retrospectively using a modified TBS Calculator (v2.2) to consider soft tissue thickness directly in the algorithm instead of BMI (Medimaps group, Plan-les-Ouates, Geneva, Switzerland), using DXA files from the original phase 2 trial. All patient identifiers were removed to maintain anonymity and to ensure blinding of the investigators to all clinical parameters and outcomes. The same region of interest as BMD was used with vertebral site exclusions as noted above. Of 222 women randomized to one of the five treatment arms, 221 received at least one dose of study medication. TBS could not be calculated in 76 patients because the DXA scanner or the acquisition mode used was incompatible with TBS software. Additional patients $(n=4)$ were excluded as a DXA change in software (upgrade to convert a QDR $4500 \mathrm{~W}$ to a Discovery $\mathrm{W}$ ) was performed in the middle of the study without TBS cross-calibration, or the DXA displayed malfunctioning detectors $(n=3)$ that could have led to errors in the TBS calculations. 


\section{Statistical analysis}

The primary outcome of interest was the change in TBS in the lumbar spine relative to baseline, assessed both by percentage change from baseline and by generalized estimating equations (GEE), adjusted for TBS, BMI, and age at baseline; treatment, visit, and treatment and visit interaction; and an unstructured covariance structure. A dose-response curve was calculated for the placebo group and 20,40 , or $80 \mu \mathrm{g}$ dose groups of abaloparatide-SC. At 24 weeks, the percentage of individuals exceeding two different levels of a priori-determined least significant change (LSC) was calculated for each group, where LSC was defined as the extent of change above which a difference in TBS could be presumed real and not secondary to precision errors. The differences in proportion of patients with an increase $\geq$ LSC were tested using the chi-square categorical test. The published precision errors for TBS vary from 1.12 to $2.1 \%$ depending on the design of the study and machine involved. The average precision across these studies was approximatively $1.53 \%$ and the corresponding LSC at $95 \%$ confidence interval was $\pm 4.2 \%[3,11,14,15]$. This latter value was used in this study. All inferential testing was twotailed, with $p \leq 0.05$ set as the threshold for statistical significance. IBM SPSS statistical software (version 21.0; SPSS, Inc.) was used for all statistics.

\section{Results}

The 138 patients included in the final analysis were generally similar to the original study population with respect to age, BMI, and lumbar spine BMD at baseline. Patients in the five treatment groups were also similar in their demographic and clinical characteristics, including baseline lumbar spine BMD and TBS measurements (Table 1).

Figure 1 shows the change in TBS over time in patients on placebo, teriparatide, and the three different doses of abaloparatide-SC. Results are expressed as percentage change (mean \pm standard error of the mean) from baseline at 12 and 24 weeks in lumbar spine TBS. After 12 weeks, lumbar spine TBS increased by $1.33,1.86,2.37$, and $1.16 \%$ in the abaloparatide-SC $20 \mu \mathrm{g}$, abaloparatide-SC $40 \mu \mathrm{g}$, abaloparatide-SC $80 \mu \mathrm{g}$, and teriparatide groups, respectively ( $p<0.05$ for all comparisons to baseline); whereas, there was no significant increase in the placebo group $(+0.23 \%, p=$ $0.35)$. After 24 weeks, TBS increased versus baseline by $2.27,3.14,4.21$, and $2.21 \%$ in the abaloparatide-SC $20 \mu \mathrm{g}$, abaloparatide-SC $40 \mu \mathrm{g}$, abaloparatide-SC $80 \mu \mathrm{g}$, and teriparatide groups, respectively ( $p<0.05$ for all comparisons to baseline); while, there was a non-significant decrease in the placebo group $(-1.08 \%, p=0.13)$.

GEE analysis, adjusted for treatment group, visit, treatment group and visit interaction, TBS at baseline, age at baseline, and BMI at baseline, showed that TBS at 12 weeks increased significantly versus placebo in patients in the abaloparatideSC $40 \mu \mathrm{g}(1.61 \%, p=0.03)$ and $80 \mu \mathrm{g}(2.07 \%, p=0.02)$ groups, but not in the abaloparatide-SC $20 \mu \mathrm{g}(1.10 \%, p=$ $0.15)$ or teriparatide $(1.00 \%, p=0.21)$ groups. No significant differences were observed between teriparatide and the abaloparatide-SC $20 \mu \mathrm{g}(0.08 \%, p=0.91)$, abaloparatide-SC $40 \mu \mathrm{g}(0.59 \%, p=0.42)$ or abaloparatide-SC $80 \mu \mathrm{g}(1.02 \%$, $p=0.24)$ groups.

At 24 weeks, all treated groups displayed significant TBS improvements versus placebo. TBS increased by $3.25 \%$ $(p<0.001)$ in the abaloparatide-SC $20 \mu \mathrm{g}$ group, $4.17 \%$ $(p<0.001)$ in the abaloparatide-SC $40 \mu \mathrm{g}$ group, $5.23 \%$ $(p<0.001)$ in the abaloparatide-SC $80 \mu \mathrm{g}$ group, and $3.27 \%$ $(p=0.002)$ in the teriparatide group. TBS also increased significantly more in patients treated with abaloparatide-SC $80 \mu \mathrm{g}(1.90 \%, p=0.04)$, but not in those treated with abaloparatide-SC $20 \mu \mathrm{g}(0.06 \%, p=0.95)$ or abaloparatideSC $40 \mu \mathrm{g}(0.86 \%, p=0.40)$ groups compared to those treated with teriparatide. Additionally, a positive dose-response was observed at 24 weeks (least square regression \pm standard error $=0.03 \pm 0.01 ; p=0.02$ ) when comparing the three different doses of abaloparatide-SC and placebo.
Table 1 Baseline characteristics of the five treatment groups expressed as mean \pm SD. No significant differences were found among groups for each reported parameter

\begin{tabular}{|c|c|c|c|c|c|c|c|c|c|c|}
\hline \multirow[t]{2}{*}{$N=138$} & \multicolumn{2}{|c|}{$\begin{array}{l}\text { ABL-SC } \\
80 \mu \mathrm{g} n=24\end{array}$} & \multicolumn{2}{|c|}{$\begin{array}{l}\text { ABL-SC } \\
40 \mu \mathrm{g} n=25\end{array}$} & \multicolumn{2}{|c|}{$\begin{array}{l}\text { ABL-SC } \\
20 \mu \mathrm{g} n=29\end{array}$} & \multicolumn{2}{|l|}{$\begin{array}{l}\text { TPTD } \\
n=31\end{array}$} & \multicolumn{2}{|c|}{$\begin{array}{l}\text { Placebo } \\
n=29\end{array}$} \\
\hline & Mean & SD & Mean & $\mathrm{SD}$ & Mean & $\mathrm{SD}$ & Mean & $\mathrm{SD}$ & Mean & SD \\
\hline Age (years) & 64.3 & 6.9 & 65.4 & 6.9 & 68.1 & 6.3 & 66.2 & 7.3 & 66.7 & 7.6 \\
\hline $\begin{array}{l}\mathrm{BMI} \\
\quad\left(\mathrm{kg} / \mathrm{m}^{2}\right)\end{array}$ & 25.6 & 3.4 & 26.1 & 3.9 & 26.4 & 2.7 & 26.7 & 3.1 & 25.5 & 3.3 \\
\hline $\begin{array}{l}\text { Lumbar spine } \\
\text { BMD } \\
\left(\mathrm{g} / \mathrm{cm}^{2}\right)\end{array}$ & 0.765 & 0.111 & 0.752 & 0.080 & 0.767 & 0.103 & 0.762 & 0.096 & 0.796 & 0.102 \\
\hline $\begin{array}{l}\text { Lumbar spine } \\
\text { TBS }\end{array}$ & 1.181 & 0.078 & 1.188 & 0.762 & 1.189 & 0.063 & 1.201 & 0.068 & 1.196 & 0.067 \\
\hline
\end{tabular}

$A B L-S C$ abaloparatide-SC, $B M D$ bone mineral density, $B M I$ body mass index, $T B S$ trabecular bone score, $T P T D$ teriparatide 


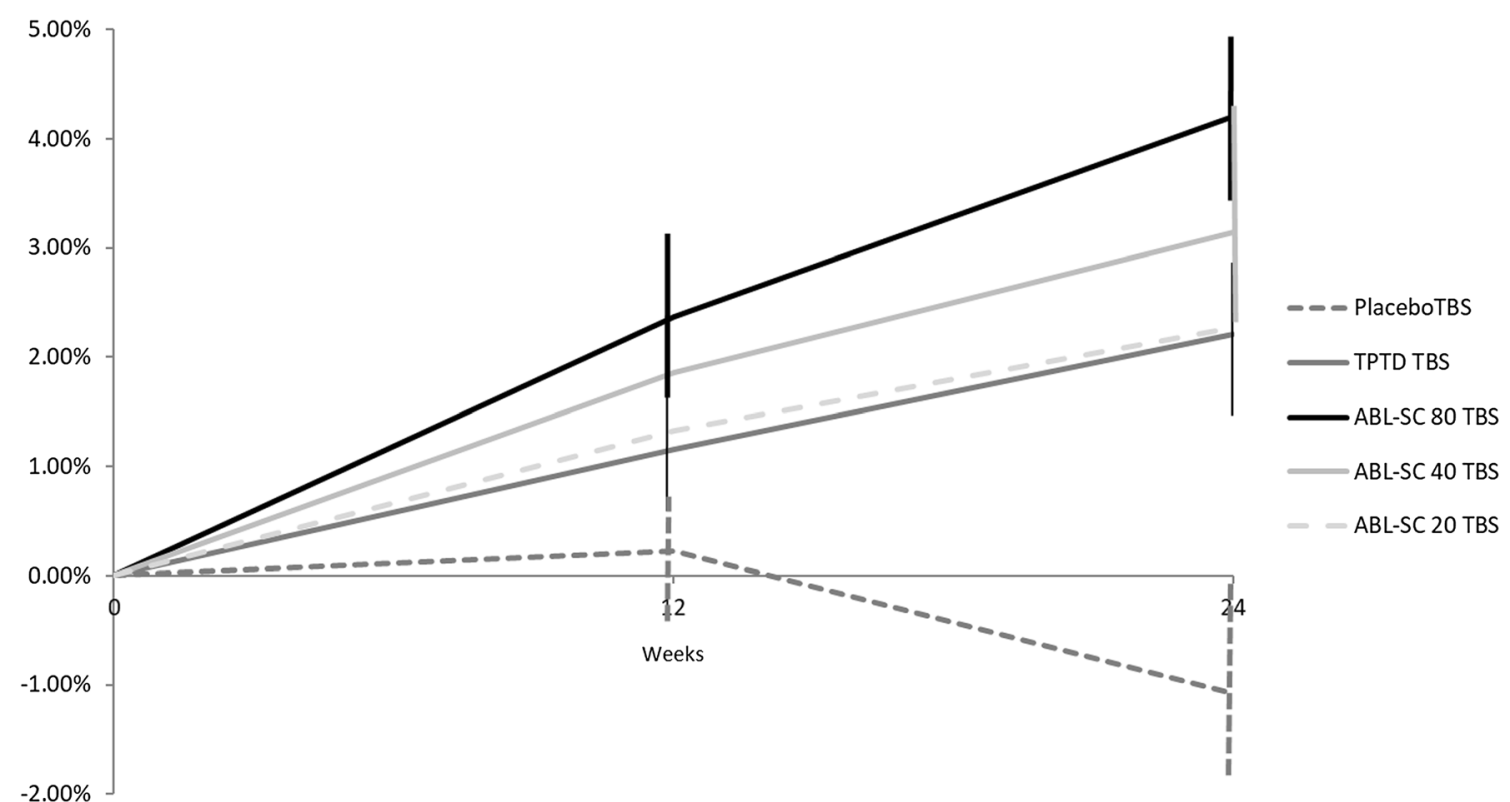

Fig. 1 Comparing change in the trabecular bone score over time in patients on placebo, teriparatide, and three different doses of abaloparatide-SC $(20,40$, and $80 \mu \mathrm{g})$. Results are expressed in percentage change (mean \pm SEM) from baseline at 12 and 24 weeks in lumbar spine TBS. TBS increases from baseline were significant for

On LSC analysis (Table 2), the percentages of patients on $80 \mu \mathrm{g}$ abaloparatide-SC daily, teriparatide $20 \mu \mathrm{g}$, and placebo who experienced greater than a $4.2 \%$ increase in TBS versus baseline were $52.2,30.0$, and $10.2 \%$, respectively. No statistical difference was found between the abaloparatide-SC $80 \mu \mathrm{g}$ and teriparatide groups $(p=0.12)$ but the $80 \mu \mathrm{g}$ abaloparatide-SC group displayed a statistically significant difference versus placebo $(p<0.01)$. Differences between the teriparatide and placebo groups were not statistically significant $(p=0.06)$. None of the patients in the abaloparatideSC $80 \mu \mathrm{g}$ group experienced a decrease in TBS versus baseline greater than the LSC, versus $6.7 \%$ of patients in the teriparatide group.

For both the teriparatide and abaloparatide-SC $80 \mu \mathrm{g}$ groups, we calculated the number of patients exceeding the LSC for lumbar spine BMD using the same $4.2 \%$ threshold as teriparatide and for all three doses of abaloparatide-SC, at 12 and 24 weeks ( $p<0.05$ for all comparisons to baseline). For placebo, there was no significant increase at 12 weeks $(p=0.35)$ and a non-significant decrease at 24 weeks $(p=0.13)$

for TBS. In the abaloparatide-SC $80 \mu \mathrm{g}$ group, 52 and $74 \%$ of patients exceeded the LSC for the lumbar spine TBS and BMD, respectively, and $35 \%$ of patients exceeded the LSC in both parameters. Results are 30 and $60 \%$ for teriparatide patients for lumbar spine TBS and BMD, respectively, and $27 \%$ for both parameters.

\section{Discussion}

Adding to the insights gained from the previously published BMD results in the same phase 2 study population [13], this analysis of the effect of abaloparatide-SC shows significant enhancement of TBS relative to placebo. Similar TBS results with teriparatide have been observed but over a longer period of time (18 to 24 months
Table 2 Least significant change (LSC) analysis comparing abaloparatide-SC $80 \mu \mathrm{g}$, teriparatide (TPTD), and placebo for two different LSC levels

\begin{tabular}{lllll}
\hline & & At 6 months & & \\
\cline { 3 - 5 } & LSC 95\% & ABL-SC 80 $\mu \mathrm{g}$ & TPTD & Placebo \\
\hline Significant individual gain (\%) & +4.20 & $52.2^{*}$ & $30.0^{\ddagger}$ & 10.3 \\
Significant individual loss (\%) & -4.20 & $0.0^{\ddagger}$ & 6.7 & 17.2 \\
\hline
\end{tabular}

$A B L-S C$ abaloparatide-SC, $L S C$ least significant change, TPTD teriparatide

$* p=0.001$ versus placebo

${ }^{\star} p=0.06$ versus placebo

${ }^{¥} p=0.05$ versus placebo 
compared to 6 months here) [3]. Moreover, at both 12 and 24 weeks, the magnitude by which TBS increased was greater in the $80-\mu \mathrm{g}$ dose group of abaloparatide-SC (the dose that was used in the subsequent larger phase 3 trial) than in the teriparatide group. Similar results were observed for the $40-\mu \mathrm{g}$ abaloparatide-SC cohort. On average, in the different treatment groups, less than $10 \%$ of the variations of the BMD were explained by the variations of TBS, confirming that both parameters are unrelated, at least in part, and represent different bone properties. Also of note, an increase in TBS greater than the LSC $(4.2 \%)$ was achieved by $52.2 \%$ of patients treated with abaloparatide-SC $80 \mu \mathrm{g}$ versus $30.0 \%$ of those treated with teriparatide. The percentage of patients on placebo exhibiting a $4.2 \%$ increase over baseline was $10.3 \%$. It is also interesting to note that $35 \%$ of the patients treated with $80 \mu \mathrm{g}$ of abaloparatide increased significantly (exceeding their LSC) both their lumbar spine BMD and TBS compared to $27 \%$ for patients treated with teriparatide.

Several relatively small studies have investigated the effect of bone-active substances on TBS [16] and suggested that TBS tends to increase with treatments that increase lumbar spine BMD and to decrease without treatment. The response usually parallels the changes observed on BMD but to a lesser extent for TBS. In contrast, the magnitude of the decrease in TBS without treatment is very similar to that of BMD. It is interesting to note that there is a different TBS response depending upon the type of treatment [16]. Usually, the antiresorptive drugs preserve bone structure, an effect supported by the mechanism of action of these agents. In these instances, monitoring TBS changes do not add substantially to what would be observed by monitoring BMD alone. However, a safety issue is relevant if TBS values were to decline significantly over time, causing concern perhaps for increased risk of fracture. In contrast, exposure to anabolic treatment is associated with an increase in TBS values above the LSC in almost two thirds of patients undergoing treatment [3]. In addition, with TBS monitoring, only $10 \%$ of patients demonstrated a decline in TBS values. In this latter case, monitoring TBS could also have clinical relevance. Whether the increase in TBS values in the context of anabolic treatment is associated with a reduction in fracture risk, over and above what an increase in BMD would indicate, remains yet to be demonstrated.

Limitations of this study include the lack of blinding of the teriparatide group. However, the TBS analysis is an objective measure and was carried out in blinded fashion without regard to the experimental arm of the study. Another limitation of this study is that it is a post hoc analysis of a phase 2 trial. The exclusion of 76 patients from the original study population is an additional limitation. However, the subgroup of analyzed patients was generally similar to the original study population with respect to several key baseline characteristics (age, BMI, and lumbar spine BMD at baseline). The patient sample also excluded women under age 55 , men of any age, and patients with secondary sources of osteoporosis, such as chronic glucocorticoid steroid use, so the results presented here cannot be extrapolated beyond the current study population.

These results help to differentiate further abaloparatide-SC from teriparatide in terms of potential effects on skeletal microarchitecture as determined indirectly by TBS, and provide additional support for the development of abaloparatide$\mathrm{SC}$ as a new anabolic treatment for postmenopausal osteoporosis.

Acknowledgements The original trial upon which these analyses are based (NCT00542425) was sponsored by Radius Health, Inc., which also provided financial support for the current study. Editorial assistance was provided by Ted Everson, $\mathrm{PhD}$, an employee of Radius Health, Inc.

\section{Compliance with ethical standards}

Conflicts of interest JPB is a consultant for Radius Health, Inc., Shire, Amgen, Eli Lilly, and Ultragenyx.

GH, LAF, AGH, are employees of Radius Health, Inc., and own company stock.

$\mathrm{KB}$ is an employee of Radius Health, Inc.

ES and JRZ have nothing to disclose.

BZL has been a consultant for Merck, and has been a consultant for and received research funding from Amgen and Eli Lilly.

DH is co-owner of the trabecular bone score (TBS) patent and is a part-time employee of Medimaps Group, and owns company stock.

Statement of informed consent All patients had provided informed written consent prior to their participation, in accordance with the Declaration of Helsinki for research on human subjects.

ABL-SC, abaloparatide-SC; SEM, standard error of the mean; TBS, trabecular bone score; TPTD, teriparatide.

Open Access This article is distributed under the terms of the Creative Commons Attribution-NonCommercial 4.0 International License (http:// creativecommons.org/licenses/by-nc/4.0/), which permits any noncommercial use, distribution, and reproduction in any medium, provided you give appropriate credit to the original author(s) and the source, provide a link to the Creative Commons license, and indicate if changes were made.

\section{References}

1. Hattersley G, Dean T, Corbin BA, Bahar H, Gardella TJ (2016) Binding selectivity of abaloparatide for PTH-type-1-receptor conformations and effects on downstream signaling. Endocrinology 157:141-149

2. Miller PD, Hattersley G, Riis BJ et al (2016) Effect of abaloparatide vs placebo on new vertebral fractures in postmenopausal women with osteoporosis. JAMA 316:722-733

3. Senn C, Gunther B, Popp AW, Perrelet R, Hans D, Lippuner K (2014) Comparative effects of teriparatide and ibandronate on spine bone mineral density (BMD) and microarchitecture (TBS) in 
postmenopausal women with osteoporosis: a 2-year open-label study. Osteoporos Int 25:1945-1951

4. Chen P, Miller PD, Recker R et al (2007) Increases in BMD correlate with improvements in bone microarchitecture with teriparatide treatment in postmenopausal women with osteoporosis. J Bone Miner Res 22:1173-1180

5. Varela A, Chouinard L, Lesage E, Smith SY, Hattersley G (2017) One year of abaloparatide, a selective activator of the PTH1 receptor, increased bone formation and bone mass in osteopenic ovariectomized rats without increasing bone resorption. J Bone Miner Res 32:24-33

6. Varela A, Haile S, Doyle N, Smith SY, Guldberg R, Hattersley G (2014) The long-term effects of abaloparatide (BA058) on micro$\mathrm{CT}$ and histomorphometry in osteopenic cynomolgus monkeys. J Bone Miner Res 29(Suppl 1):S18

7. Doyle N, Varela A, Smith SY, Hattersley G (2014) Abaloparatide (BA058), a human PTHrP analog: correlation of in vivo bone mass gains and improved strength in the osteopenic cynomolgus monkey. J Bone Miner Res 29:S319

8. Bahar H, Doyle N, Varela A, et al. (2015) Abaloparatide treatment increases bone formation without corresponding increases in bone resorption resulting in marked bone gains in osteopenic ovariectomized monkeys and rats. Endoc Rev 36:Abstract OR08-3

9. Hans D, Barthe N, Boutroy S, Pothuaud L, Winzenrieth R, Krieg MA (2011) Correlations between trabecular bone score, measured using anteroposterior dual-energy X-ray absorptiometry acquisition, and 3-dimensional parameters of bone microarchitecture: an experimental study on human cadaver vertebrae. J Clin Densitom $14: 302-312$

10. Muschitz C, Kocijan R, Haschka J et al (2015) TBS reflects trabecular microarchitecture in premenopausal women and men with idiopathic osteoporosis and low-traumatic fractures. Bone 79:259-266

11. Silva BC, Leslie WD, Resch H et al (2014) Trabecular bone score: a noninvasive analytical method based upon the DXA image. J Bone Miner Res 29:518-530

12. McCloskey EV, Odén A, Harvey NC et al (2016) A meta-analysis of trabecular bone score in fracture risk prediction and its relationship to FRAX. J Bone Miner Res 31:940-948

13. Leder BZ, O'Dea LS, Zanchetta JR et al (2015) Effects of abaloparatide, a human parathyroid hormone-related peptide ana$\log$, on bone mineral density in postmenopausal women with osteoporosis. J Clin Endocrinol Metab 100:697-706

14. Harvey NC, Glüer CC, Binkley N et al (2015) Trabecular bone score (TBS) as a new complementary approach for osteoporosis evaluation in clinical practice. Bone 78:216-224

15. Silva BC, Broy SB, Boutroy S, Schousboe JT, Shepherd JA, Leslie WD (2015) Fracture risk prediction by non-BMD DXA measures: the 2015 ISCD official positions part 2: trabecular bone score. J Clin Densitom 18:309-330

16. Shevroja E, Lamy O, Kohlmeier L, Koromani F, Rivadeneira F, Hans D (2017) Use of trabecular bone score (TBS) as a complementary approach to dual-energy x-ray absorptiometry (DXA) for fracture risk assessment in clinical practice. J Clin Densitom 20: 334-345 